\title{
THE INFLUENCE OF CELL WINDOW IMPERFECTIONS ON THE CALIBRATION AND MEASURED DATA OF TWO TYPES OF ROTATING-ANALYZER ELLIPSOMETERS
}

\author{
A. STRAAIJER, L.J. HANEKAMP and G.A. BOOTSMA \\ Department of Applied Physics, Twente University of Technology, P.O. Box 217, 7500 AE \\ Enschede, The Netherlands
}

Received 20 August 1979

A graphical method has been developed to determine the plane of incidence in the presence of cell windows with small retardation. For two types of rotating-analyzer ellipsometers, expressions have been derived that relate the experimental parameters and the elements of the Mueller imperfection matrices of the windows. These matrices can be determined by measuring with and without cell windows. Measurements have been performed with three samples with different optical constants.

\section{Introduction}

In the last ten years several types of automatic ellipsometers have been described. In his recent review Muller [1] distinguishes two groups, with and without compensation of the elliptic polarization. The group of non-compensating ellipsometers may be subdivided in subgroups with different modulation principles. The polarizer-sample-rotating analyzer $\left(P S A_{\mathrm{rot}}\right)$ and the polarizer-sample-rotating analyzer-fixed analyzer $\left(P S A_{\mathrm{rot}} A\right)$ ellipsometers belong to the subgroup with a rotating analyzer. The first measures phase and irradiance (intensity method) $[2,3]$, the second two phases (phase method) [4]. The effect of azimuth-angle errors, component and cell window imperfections on the ellipsometric parameters has been reviewed by Azzam and Bashara [5]. For the polarizer-compensator-sampleanalyzer (PCSA) ellipsometer a number of these errors can be eliminated by averaging of two- or four-zone measurements. An averaging procedure can also be applied to rotating-analyzer ellipsometers by introducing different measuring modes. However, the effect of birefringence of the cell windows cannot be eliminated in this way. A general theoretical treatment of cell window birefringence and an experimental method to correct for this effect with the PCSA ellipsometer have also been presented [5]. An analysis of systematic errors in rotating-analyzer ellipsometers has been given by the same authors [6]. Some earlier measurements with a $P S A_{\text {rot }}$ ellipsometer were strongly influenced by window birefringence [7]. 
The present paper describes a graphical method of determining the plane of incidence in the presence of cell windows with small retardation. In this case the cell windows can be treated as small-retardation wave-plates (SRWP). This method makes it possible also to calculate half of the elements of the imperfection matrices describing the birefringence of the cell windows. The complete imperfection matrices can be determined with the $P S A_{\text {rot }}$ as well as the $P S A_{\text {rot }} A$ ellipsometer from the shift in the ellipsometric parameters by measuring with and without cell windows.

\section{Experimental}

\subsection{Ellipsometers}

A schematic diagram of the two ellipsometers of the rotating-analyzer type that have been built in our laboratory is given in fig. 1 . The set-up of the $P S A_{\text {rot }}$ type can be easily changed into the $P S A_{\text {rot }} A$ configuration by introducing a fixed analyzer between the rotating analyzer and the detector without changing the positions of the other components. The rotating analyzer is speed-controlled [4] with an accuracy within $5 \mathrm{ppm}$ at an angular frequency of $66.6 \mathrm{~Hz}$. When using the intensity method, the periodic analog detector signal is converted into digital form on sampling instants given by a general timing circuit $[2,3]$. In a special computer program these converted intensities are averaged and Fourier-analyzed and the

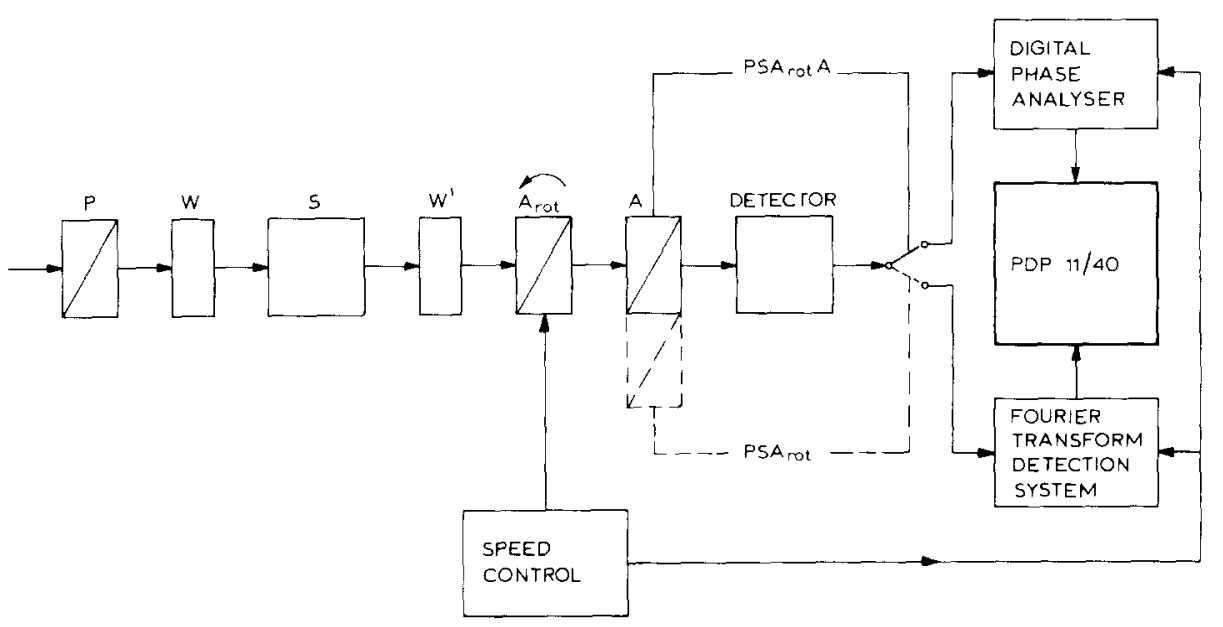

Fig. 1. Schematic diagram of the $P S A_{\text {rot }}$ and $P S A_{\text {rot }} A$ ellipsometer. By introducing an analyzer in the configuration and by switching the detector signal to another electronic device, the $P S A_{\text {rot }}$ ellipsometer is changed into the $P S A_{\text {rot }} A$ configuration. 
ellipsometric $\Delta$ and $\psi$ values are calculated from the two Fourier coefficients, $a$ and $b$. With the phase method, the harmonic detector signal is fed into a filter circuit where two frequencies are separated as described in refs. $[4,8]$. The phases $\gamma_{2 \omega}$ and $\gamma_{4 \omega}$ of the two frequencies are measured and displayed. From these phases the ellipsometric $\Delta$ and $\psi$ values are calculated.

For the ideal case of the intensity ellipsometer, formulas for the calculation of $\Delta$ and $\psi$ from the two Fourier coefficients $a$ and $b$ have been presented in ref. [2]. More complicated expressions, taking into account small polarizer imperfections, the calibration azimuth of $A_{\text {rot }}$, and errors introduced by using a low-pass filter have been developed by Aspnes [9]. We have applied different operation modes with different angles of the fixed polarizer $\left(P=0^{\circ}, 90^{\circ}\right.$ and $\left.\pm 45^{\circ}\right)$. In the calibration mode $\left(P=0^{\circ}, 90^{\circ}\right)$ no information about the reflecting surface is contained in the Fourier coefficients, $a_{0}$ and $b_{0}$, but only about the mounting position of $A_{\text {rot }}$. The measuring mode $\left(P= \pm 45^{\circ}\right)$ yields the Fourier coefficients $a$ and $b$ which are used to calculate $\Delta$ and $\psi$ for the reflecting surface. To overcome time-consuming trigonometric calculations the following expressions were developed

$\cos 2 \psi=-\left(a_{0} a+b_{0} b\right) /\left(a_{0}^{2}+b_{0}^{2}\right)$,

$\cos \Delta= \pm\left(a_{0} b-a b_{0}\right)\left\{\left(a_{0}^{2}+b_{0}^{2}\right)-\left(a_{0} a+b_{0} b\right)^{2}\right\}^{1 / 2}$.

The upper and lower sign in eq. (1b) represent $P=+45^{\circ}$ and $P=-45^{\circ}$, respectively. For an accurate measurement of $\Delta$ and $\psi$ it proved to be necessary to average these values for the two symmetric settings $P= \pm 45^{\circ}$.

A similar procedure was followed for the phase ellipsometer. The phases $\gamma_{2 \omega}^{0}$ and $\gamma_{4 \omega}^{0}$ in the calibration mode $\left(P=A=0^{\circ}\right)$ contain no information about the reflecting surface but they are generated by the mounting position of $A_{\text {rot }}$ and by the electronics. To attain optimal noise characteristics (cf. ref. [8]) and the best symmetry in the measuring mode, we selected eight combinations of $P$ and $A$. In analogy with ref. [10] these combinations are called "zones". The definitions for these zones and the expressions for $\Delta$ and $\psi$ are given in table 1 . In this table $T_{2}=$ $\tan \left(\gamma_{2 \omega}-\gamma_{2 \omega}^{0}\right)$ and $T_{4}=\tan \left(\gamma_{4 \omega}-\gamma_{4 \omega}^{0}\right)$. The phases $\gamma_{2 \omega}$ and $\gamma_{4 \omega}$ pertain to the measuring mode. For an accurate determination of $\Delta$ and $\psi$ it proved to be

Table 1

Relations between $\Delta$ and $\psi$ and the measured phases; upper signs hold for $P=-45^{\circ}$ (odd zones), lower for $P=+45^{\circ}$ (even zones)

\begin{tabular}{lllll}
\hline$A(\mathrm{deg})$ & 0 & +45 & +90 & -45 \\
Zone & 1,2 & 3,4 & 5,6 & 7,8 \\
\hline $\cos \Delta$ & $\pm T_{4} / \tan 2 \psi$ & $\mp\left(T_{4} \tan 2 \psi\right)^{-1}$ & $\pm T_{4} / \tan 2 \psi$ & $\mp\left(T_{4} \tan 2 \psi\right)^{-1}$ \\
$\cos 2 \psi$ & $T_{2} /\left(T_{2}-T_{4}\right)$ & $-T_{4} /\left(T_{2} T_{4}+1\right)$ & $-T_{2} /\left(T_{2}-T_{4}\right)$ & $T_{4} /\left(T_{2} T_{4}+1\right)$ \\
\hline
\end{tabular}


necessary to average at least over two zones, preferably over a zone pair with $P=$ $\pm 45^{\circ}$.

\subsection{Windows and samples}

For the investigation of changes in the Fourier coefficients or in the phases caused by cell windows, two birefringent glass windows were reproducibly placed into and out of the optical arrangement. The windows were of pyrex glass with a diameter of $15 \mathrm{~mm}$ and a thickness of about $2.5 \mathrm{~mm}$ and were fixed to a stainless steel vacuum flange.

Because the influence of glass windows depends considerably on the $\Delta$ and $\psi$ value of the reflecting surface (cf. section 3), three samples with different optical constants were taken. These were a polished stainless steel surface, a silicon wafer with a Langmuir Blodgett layer of cadmium stearate of about $590 \AA$, and a gold layer covered with an undefined film on a glass substrate (table 6).

\section{Theory}

Recently the problem of component imperfections has been approached with the Mueller matrix theory $[11,12,7]$, where the state of polarization of a light beam is described with the $4 \times 1$ Stokes column vector. The advantage of this calculus is that the vector elements represent real quantities and that the first element represents the light intensity. The optical components $(k)$ are described by $4 \times 4$ matrices $\boldsymbol{T}_{k}=\boldsymbol{T}_{k}^{0}+\delta \boldsymbol{T}_{k}$, where $\boldsymbol{T}_{k}^{0}$ is the ideal component matrix and $\delta \boldsymbol{T}_{k}$ is the matrix which contains all component imperfections.

In this paper we restrict ourselves to window imperfections. To simplify the calculations we only consider small window birefringence (SRWP approximation) and abandon possible dichroism. The Mueller imperfection matrix, proposed in ref. [11] for this approximation appears to be a good choice (see section 4).

\subsection{Plane of incidence}

The extinction criterion for the PCSA ellipsometer is that the derivative of the intensity of the light flux impinging on the detector with respect to the azimuth of the polarizers $(P, A)$ should be zero. Generally for every kind of component imperfections a matrix of coupling coefficients can be derived of which every element Inust be multiplied with the elements of the corresponding imperfection matrix $\delta \boldsymbol{T}_{k}$. Summation of these products yields the theoretical deviations in $P$ and $A$ from extinction, as shown in refs. [12,5].

This method of calculation is used in this paper to investigate the influence of birefringent glass windows on the determination of the plane of incidence. The arrays of coupling coefficients are derived for two windows in the calibration con- 
figuration of the polarizer-entrance window-sample-exit window-analyzer $\left(P W S W^{\prime} A\right)$ ellipsometer. In the calculation of the deviations in $P$ and $A$ use is made of:

(i) Stokes column vectors $\boldsymbol{S}_{k I}$ and $\boldsymbol{S}_{k \mathrm{O}}$, horizontally written between curly brackets, describing the polarization states of the light flux before and after passing component $k$, with $k$ being $P, W, S, W^{\prime}$ or $A$;

(ii) Mueller matrix $\boldsymbol{T}_{k}$ of the component $k$;

(iii) row vectors $\Gamma_{k}$, written between square brackets, determining the light flux contribution due to Stokes-vector perturbations generated at the output of the $k$ th component due to its imperfection.

The intensity of the light flux falling on the detector $\left(I_{\mathrm{D}}\right)$ is a function of variations in component properties and of changed Stokes vectors. Restricting ourselves to window corrections, we find that application of the extinction conditions,

$\partial I_{\mathrm{D}} / \partial P=0, \quad \partial I_{\mathrm{D}} / \partial A=0$,

yields respectively

$\delta P\left(-\Gamma_{P}^{0} \frac{\partial^{2} \boldsymbol{S}_{P 0}}{\partial P^{2}}\right)+\delta A\left(-\frac{\partial \boldsymbol{\Gamma}_{P}}{\partial A} \frac{\partial \boldsymbol{S}_{P 0}}{\partial P}\right)=\sum_{k=W, W^{\prime}} \Gamma_{k}^{0} \delta \boldsymbol{T}_{k} \frac{\partial \boldsymbol{S}_{k I}}{\partial P}$,

$\delta P\left(-\frac{\partial \boldsymbol{\Gamma}_{P}}{\partial A} \frac{\partial \boldsymbol{S}_{P 0}}{\partial A}\right)+\delta A\left(-\frac{\partial^{2} \boldsymbol{\Gamma}_{P}}{\partial A^{2}} \boldsymbol{S}_{P 0}^{0}\right)=\sum_{k=W, W^{\prime}} \frac{\partial \boldsymbol{\Gamma}_{k}}{\partial A} \delta \boldsymbol{T}_{k} \boldsymbol{S}_{k \mathrm{I}}^{0}$.

Here $\Gamma_{P}^{0}=\Gamma_{W}^{0}=\Gamma_{A}^{0} \boldsymbol{T}_{S}^{0} \boldsymbol{T}_{A}^{0}, \boldsymbol{\Gamma}_{S}^{0}=\Gamma_{W^{\prime}}^{0}=\Gamma_{A}^{0} \boldsymbol{T}_{A}^{0}, \boldsymbol{\Gamma}_{A}^{0}=[1,0,0,0]$ and $\boldsymbol{T}_{W}^{0}=\boldsymbol{T}_{W^{\prime}}^{0}=1$. $\boldsymbol{S}_{k}^{0}, \boldsymbol{\Gamma}_{k}^{0}$ and $\boldsymbol{T}_{k}^{0}$ point to the situation without imperfections. The results of the calculation of the unknown vectors in eqs. (3) and (4) are presented in table 2 . The lefthand sides of these equations contain the variables $\delta P$ and $\delta A$ and the right-hand sides are constant. They contain individual elements $\left(t_{i j}\right)_{k}$ of the unknown window imperfection matrices $\delta T_{k}$. The straight lines in a $P$-versus- $A$ representation correspond to the "fixing $P$ " and "fixing $A$ " lines in the alignement procedure (cf. section 4.1). From eqs. (3) and (4) the slopes $(m)$ of the lines are calculated to be

$$
\begin{aligned}
& m_{1,3}=(\sin 2 \psi \cos \Delta) /(1 \mp \cos 2 \psi), \\
& m_{2,4}=(1 \pm \cos 2 \psi) /(\sin 2 \psi \cos \Delta) .
\end{aligned}
$$

For a definition of the $m$ 's, see fig. 2 .

By solving eqs. (3) and (4), $\delta P$ and $\delta A$ can be expressed in terms of the elements $\left(t_{i j}\right)_{k}$, in general form:

$$
\begin{aligned}
& \delta P=\sum_{i, j} \beta_{i j}(W)\left(t_{i j}\right)_{W}+\sum_{i, j} \beta_{i j}\left(W^{\prime}\right)\left(t_{i j}\right)_{W^{\prime}}, \\
& \delta A=\sum_{i, j} \alpha_{i j}(W)\left(t_{i j}\right)_{W}+\sum_{i, j} \alpha_{i j}\left(W^{\prime}\right)\left(t_{i j}\right)_{W^{\prime}}
\end{aligned}
$$

The coupling coefficients $\alpha_{i j}$ and $\beta_{i j}$ are given in table 3 . The imperfection matrix 
Table 2

Column and row vectors in eqs. (3) and (4) for the extinction settings of $P$ and $A$ (cf, ref, [12], tables 1 and 2$)$; upper signs are for case $1\left(P=0^{\circ}, A=90^{\circ}\right)$, lower for case $2\left(P=90^{\circ}, A=0^{\circ}\right)$; the abbreviations $s_{\theta}$ and $c_{\theta}$ are used for sine and cosine of $\theta$

\begin{tabular}{|c|c|c|}
\hline Element $k$ & $s_{k \mathrm{I}}^{0}$ & $s_{k I}^{0}(1,2)$ \\
\hline$P$ & $\{1,0,0, \mu\}$ & $\{1,0,0, \mu\}$ \\
\hline$W, S$ & $\left\{1, c_{2 p}, s_{2} p, 0\right\}$ & $\{1, \pm 1,0,0\}$ \\
\hline \multirow[t]{2}{*}{$W^{\prime}, A$} & $\left\{1-c_{2 \psi} c_{2 P}, c_{2 P}-c_{2 \psi}, s_{2} p s_{2 \psi} c_{\Delta},-s_{2} p s_{2 \psi} s_{\Delta}\right\}$ & $\left\{1 \mp c_{2 \psi}, \pm 1-c_{2 \psi}, 0,0\right\}$ \\
\hline & $\partial S_{k 1}^{0} / \partial P$ & $\partial S_{k 1}^{0} / \partial P(1,2)$ \\
\hline$P$ & $\{0,0,0,0\}$ & $\{0,0,0,0\}$ \\
\hline$w, s$ & $\left\{0,-2 s_{2 P}, 2 c_{2 P}, 0\right\}$ & $\{0,0, \pm 2,0\}$ \\
\hline$W^{\prime \prime}, A$ & 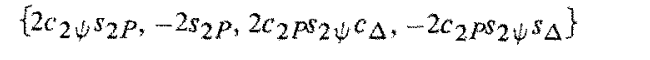 & $\left\{0,0, \pm 2 s_{2 \psi} c_{\Delta}, \mp 2 s_{2 \psi} s_{\Delta}\right\}$ \\
\hline Klement $k$ & $r_{k}^{0}$ & $\Gamma_{k}^{0}(1,2)$ \\
\hline \multirow{4}{*}{$\begin{array}{l}A \\
W^{\prime}, S \\
W, P\end{array}$} & {$[1,0,0,0]$} & {$[1,0,0,0]$} \\
\hline & {$\left[1, c_{2 A}, s_{2 A}, 0\right]$} & {$[1, \mp 1,0,0]$} \\
\hline & {$\left[1-c_{2 A} c_{2 \psi}, c_{2 A}-c_{2 \psi}, s_{2 A} s_{2 \psi} c_{\Delta}, s_{2 A} s_{2 \psi} s_{\Delta}\right]$} & {$\left[1, \pm c_{2 \psi}, \mp 1 \cdots c_{2 \psi}, 0,0\right]$} \\
\hline & $\partial \Gamma_{k}^{0} / \partial A$ & $\partial \Gamma_{k}^{0 / \partial A}(1,2)$ \\
\hline$A$ & {$[0,0,0,0]$} & {$[0,0,0,0]$} \\
\hline$W^{\prime}, s$ & {$\left[0,-2 s_{2 A}, 2 c_{2 A}, 0\right]$} & {$[0,0, \pm 2,0]$} \\
\hline$W, P$ & {$\left[2 s_{2 A} c_{2 \psi},-2 s_{2 A}, 2 c_{2 A} s_{2 \psi} c_{\Delta}, 2 c_{2 A} s_{2 \psi} s_{\Delta}\right]$} & {$\left[0,0, \mp 2 s_{2 \psi} c_{\Delta}, \mp 2 s_{2 \psi} s_{\Delta}\right]$} \\
\hline
\end{tabular}
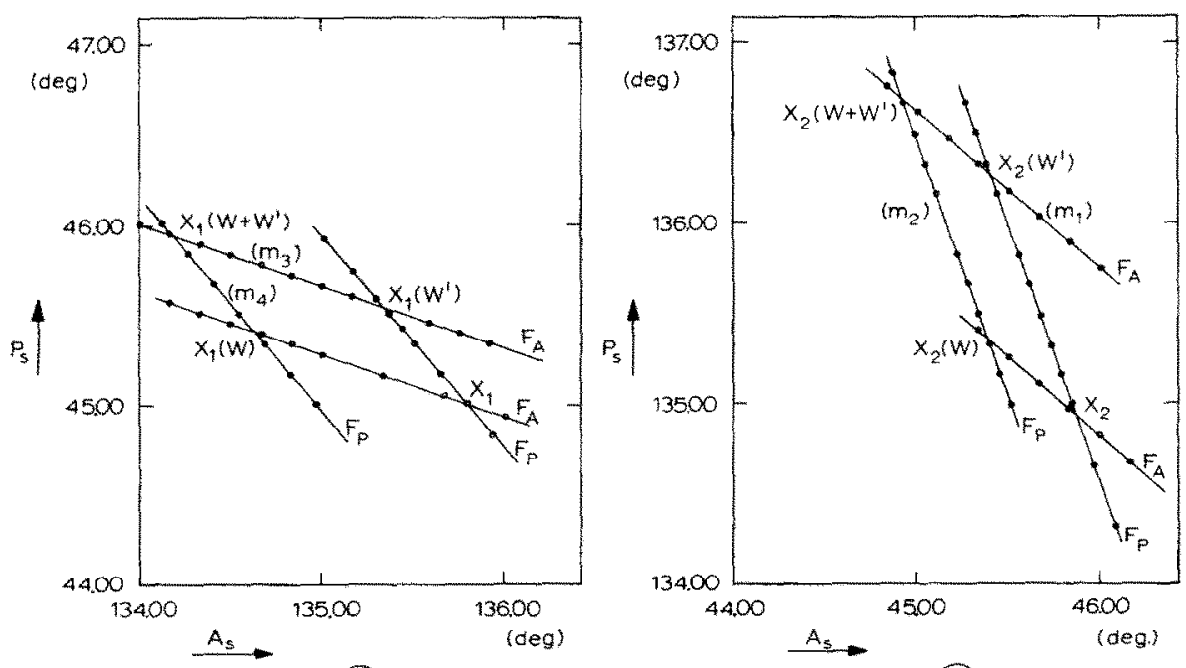

(a)

(b)

Fig. 2. $P$ and $A$ values at minimum light flux transmission for fixed $A$ and $P$ values, $F_{A}$ and $F_{P}$ (a) case 1: $P \approx 0^{\circ}, A \approx 90^{\circ}$ (b) case 2: $P \approx 90^{\circ}, A \approx 0^{\circ}, P_{\mathrm{s}}$ and $A_{s}$ indicate scale values. The points of intersection $\mathrm{X}, \mathrm{X}(W), \mathrm{X}\left(W^{\prime}\right)$ and $\mathrm{X}\left(W+W^{\prime}\right)$ represent measurements without windows, with entrance window, with exit window, and with both windows, respectively. The slopes of the lines from the measurements with both windows are $m_{1}, m_{2}, m_{3}$ and $m_{4}$. 


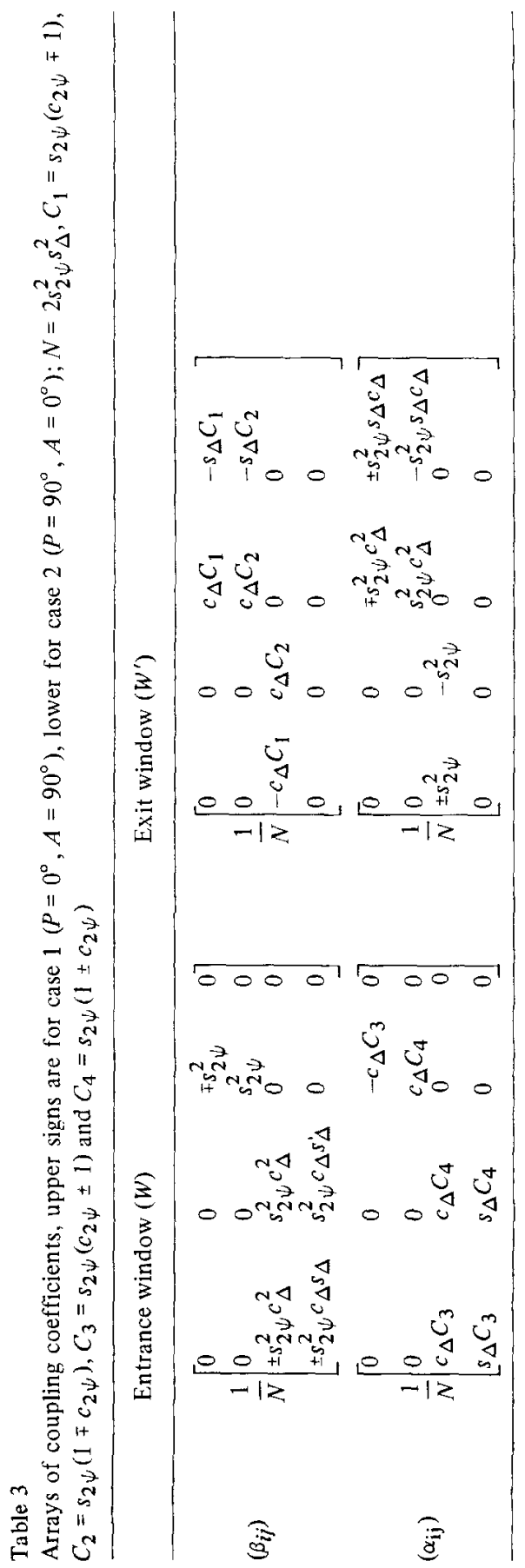


for the windows in the SRWP approximation is given by

$\delta T_{k}=\left[\begin{array}{cccc}0 & 0 & 0 & 0 \\ 0 & 0 & 0 & -B_{k} \\ 0 & 0 & 0 & A_{k} \\ 0 & B_{k} & -A_{k} & 0\end{array}\right]$.

Here $A_{k}=\delta_{k} \cos 2 \phi_{k}$ and $B_{k}=\delta_{k} \sin 2 \phi_{k}$ where $\phi_{k}$ is the azimuth and $\delta_{k}$ the retardation of the "wave plate" $k=W, W^{\prime}[11]$.

After substituting the elements of the imperfection matrices and the coupling coefficients from table 3 in eqs. (7a) and (7b) we get

$\delta P(-1 \mp \cos 2 \psi)+\delta A \sin 2 \psi \cos \Delta=-\frac{1}{2} B_{W^{\prime}} \sin 2 \psi \sin \Delta$,

$\delta P \sin 2 \psi \cos \Delta+\delta A(-1 \pm \cos 2 \psi)=-\frac{1}{2} B_{w} \sin 2 \psi \sin \Delta$.

\subsection{Rotating-analyzer ellipsometers with window influences}

In this section we calculate the influence of birefringent cell windows on the calibration and measured data of the intensity and phase ellipsometer. To get manageable results the SRWP approximation is used here also. In the calculations for both types of ellipsometers, terms containing a product of elements of the window transformation matrices are neglected. It may be remarked that this approximation cannot be applied if the ellipsometric parameters have particular values, e.g. $\Delta \approx 0^{\circ}, 90^{\circ}$ and $\psi \approx 0^{\circ}, 45^{\circ}$. For these values the neglected terms are not small with respect to the trigonometric terms containing $\Delta$ and/or $2 \psi$.

\subsubsection{Intensity ellipsometer}

For the $P W S W^{\prime} A_{\text {rot }}$ configuration the detected light intensity follows from the calculated Stokes vector of the light flux on the detector

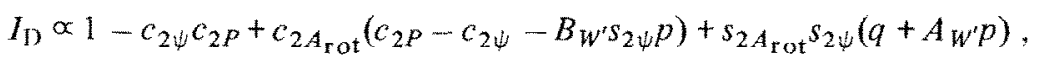

where

$p=-\sin 2 P \sin \Delta+B_{W} \cos 2 P \cos \Delta-A_{W} \sin 2 P \cos \Delta$,

$q=\sin 2 P \cos \Delta+B_{W} \cos 2 P \sin \Delta-A_{W} \sin 2 P \sin \Delta$.

This corresponds with the general representation of the intensity given by Budde [13]

$I_{\mathrm{D}} \propto k_{\mathrm{o}}\left(1+a \cos 2 A_{\mathrm{rot}}+b \sin 2 A_{\mathrm{rot}}\right)$,

where $k_{0}$ is a constant and $a$ and $b$ are the Fourier coefficients.

In table 4 the Fourier coefficients are given for calibration as well as measuring 
Table 4

The Fourier coefficients $a$ and $b$ for different modes, measured with the intensity ellipsometer

\begin{tabular}{|c|c|c|c|}
\hline $\begin{array}{l}P \\
(\text { deg })\end{array}$ & Mode & $a$ & $b$ \\
\hline $\begin{array}{r}0 \\
90\end{array}$ & $\begin{array}{l}\text { Calibration } \\
\text { Calibration }\end{array}$ & $\begin{array}{r}1 \\
-1\end{array}$ & $\begin{array}{l}s_{2 \psi} s_{\Delta} B_{W} /\left(1 \quad c_{2 \psi}\right) \\
-s_{2 \psi} s_{\Delta} B_{W} /\left(1+c_{2 \psi}\right)\end{array}$ \\
\hline $\begin{array}{l}-45 \\
+45\end{array}$ & $\begin{array}{l}\text { Measuring } \\
\text { Measuring }\end{array}$ & $\begin{array}{r}-c_{2 \psi}-s_{2 \psi} s_{\Delta} B_{W^{\prime}} \\
c_{2 \psi}+s_{2 \psi} s_{\Delta^{B}} W_{W^{\prime}}\end{array}$ & $\begin{array}{l}-s_{2 \psi} c_{\Delta}+s_{2 \psi} s_{\Delta}\left(A_{W}+A_{W^{\prime}}\right) \\
s_{2 \psi} c_{\Delta}-s_{2 \psi} s_{\Delta}\left(A_{W}+A_{W^{\prime}}\right)\end{array}$ \\
\hline
\end{tabular}

modes. For several settings of $P$, the Fourier coefficients have very simple forms. With ideal windows $\left(\delta \boldsymbol{T}_{k}=0\right), a$ and $b$ contain no information about the reflecting surface for $P=0^{\circ}$ or $90^{\circ}$ in the calibration modes. In these modes only the entrance window affects the measurement, while in the measuring modes the main influence comes from the exit window. The influence can be eliminated in the coefficient $a$, by averaging over the measuring modes.

By measuring $b$ in the calibration mode the matrix element $B_{W}$ can be directly determined (table 4). The differences in the Fourier coefficients $a$ and $b$ with and without windows in the measuring modes yield the matrix elements $B_{W^{\prime}}, A_{W}$ and $A_{W^{\prime}}$. In practice the values of $a$ and $b$ in the calibration modes without windows differ from the theoretical values \pm 1 and 0 . This is caused by the arbitrary mounting of the rotating analyzer in the turning motor shaft with respect to the optical encoder reference: the actually measured Stokes vector is rotated over a fixed angle. The elements of the Mueller matrix describing this rotation can be deter. mined by equating the calculated and measured elements of the Stokes vector $\left\{1, a, b,\left(1-a^{2}-b^{2}\right)^{1 / 2}\right\}$. All the measured Fourier coefficients have to be corrected by using this rotation matrix.

\subsubsection{Phase ellipsometer}

For the $P W S W^{\prime} A_{\text {rot }} A$ configuration the intensity of the detected light flux is calculated to be:

$$
\begin{aligned}
& I_{\mathrm{D}} \propto k+l c_{2 A_{\mathrm{rot}}}+m s_{2 A_{\mathrm{rot}}}+c_{2 A}\left\{k c_{2 A_{\mathrm{rot}}}+\frac{1}{2} l\left(c_{4 A_{\mathrm{rot}}}+1\right)+\frac{1}{2} m s_{4 A_{\mathrm{rot}}}\right\} \\
& \quad+s_{2 A}\left\{k s_{2 A_{\mathrm{rot}}}-\frac{1}{2} m\left(c_{4 A_{\mathrm{rot}}}-1\right)+\frac{1}{2} l_{4 A_{\mathrm{rot}}}\right\}
\end{aligned}
$$

where

$$
\begin{aligned}
& k=1-c_{2 \psi} c_{2 P}, \\
& l=c_{2 P}-c_{2 \psi}-B_{W^{\prime} s_{2 \psi}}\left(-s_{2 P} s_{\Delta}+c_{2 P} c_{\Delta} B_{W}-s_{2 P} c_{\Delta} A_{W}\right), \\
& m=\left\{s_{2 P}\left(c_{\Delta}-A_{W^{\prime} s_{\Delta}}\right)+\left(c_{2 P} B_{W}-s_{2 P} A_{W}\right)\left(s_{\Delta}+s_{\Delta} A_{W^{\prime}}\right)\right\} s_{2 \psi} .
\end{aligned}
$$




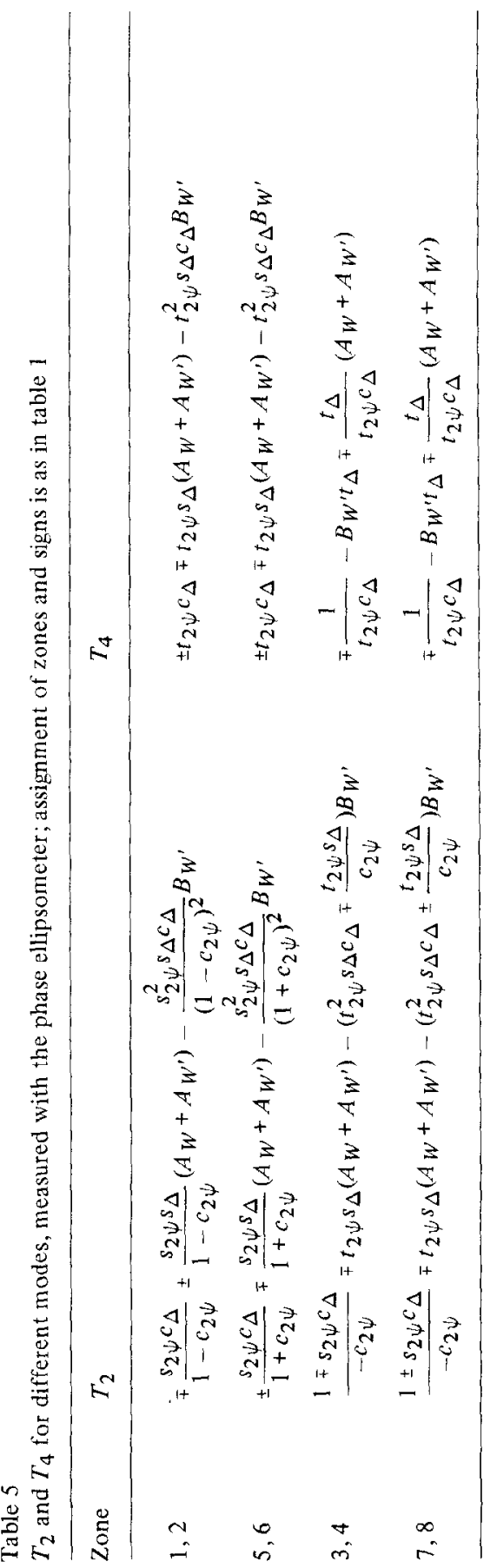


This corresponds with the general representation of the intensity

$I_{\mathrm{D}} \propto A_{0}\left(A_{1}+A_{2} \sin 2 A_{\mathrm{rot}}+A_{3} \cos 2 A_{\mathrm{rot}}+A_{4} \sin 4 A_{\mathrm{rot}}+A_{5} \cos 4 A_{\mathrm{rot}}\right)$.

The phase angles of the two frequency components in the intensity are defined by [8]

$T_{2}=\tan \gamma_{2 \omega}=A_{2} / A_{3}$,

$T_{4}=\tan \gamma_{4 \omega}=A_{4} / A_{5}$.

For the calibration modes we get

$T_{2}=\frac{1}{2} T_{4}=\frac{1}{2}\left(B_{W} \sin 2 \psi \sin \Delta\right) /(1 \mp \cos 2 \psi)$,

where the upper sign represents $P, A=0^{\circ}$ and the lower $P, A=90^{\circ}$. Without cell windows $T_{2}=T_{4}=0$ and thus the phase angles contain no information about the reflecting surface. In table $5, T_{2}$ and $T_{4}$ are given for eight different measuring modes. Substituting $B_{W}=B_{W^{\prime}}=A_{W}=A_{W^{\prime}}=0$ yields $T_{2}$ and $T_{4}$ for ideal windows. The results show that in the calibration modes errors are due to the entrance window only, while in the measuring modes most errors arise from the exit window. It appears to be impossible to remove the window errors by averaging over zones.

\section{Results and discussion}

\subsection{Plane of incidence with window influences}

The alignment procedure of McCrackin et al. [10] to determine the plane of incidence leads to incorrect results with birefringent windows. In this section we describe a procedure to eliminate the errors.

For the settings $P \approx 0^{\circ}, A \approx 90^{\circ}$ (case 1) and $P \approx 90^{\circ}, A \approx 0^{\circ}$ (case 2) the $P$ and $A$ values for minimum light-flux transmission were determined for fixed $A$ and $P$ values. This was done without windows, with entrance or exit window and with

Table 6

Ellipsometric parameters $\Delta$ and $\psi$, coordinates $P_{\mathrm{s}}$ and $A_{\mathrm{s}}$ of $\mathrm{X}_{1}$ and $\mathrm{X}_{2}$ (fig. 2) and calculated values of $P_{0}$ and $A_{0}$ for the three different samples

\begin{tabular}{lcccccccc}
\hline Sample & $\Delta$ & $\psi$ & $P_{\mathrm{s}}\left(\mathrm{X}_{1}\right)$ & $A_{\mathrm{s}}\left(\mathrm{X}_{1}\right)$ & $P_{\mathrm{s}}\left(\mathrm{X}_{2}\right)$ & $A_{\mathrm{s}}\left(\mathrm{X}_{2}\right)$ & $P_{0}$ & $A_{0}$ \\
\hline $1 \begin{array}{c}\text { Stainless } \\
\text { steel }\end{array}$ & 124.0 & 32.7 & 45.00 & 135.80 & 134.95 & 45.87 & 44.98 & 45.87 \\
$2 \begin{array}{c}\text { Silicon with } \\
\text { stearate }\end{array}$ & 92.5 & 26.7 & 44.99 & 135.81 & 134.93 & 45.84 & 44.98 & 45.84 \\
3 Gold film & 111.1 & 43.4 & 44.98 & 135.81 & 134.97 & 45.84 & 44.90 & 45.70 \\
\hline
\end{tabular}


both windows. For sample 1 (table 6 ) the results are presented in fig. 2 . The results obtained for the other samples are essentially the same. Three conclusions can be drawn:

(i) The shifts $\delta P$ and $\delta A$ caused by window imperfections are additive, in agreement with eqs. (7a) and (7b).

(ii) The introduction of the entrance window causes only a parallel shift of the $F_{F}$ line, and of the exit window only a parallel shift of the $F_{A}$ line, in agreement with eqs. (9a) and (9b). The $F_{P}$ and $F_{A}$ lines are defined in fig. 2 . In accordance with eqs. (5) and (6) the slopes of the lines appear to be independent of window imperfections.

(iii) The influence of the entrance window on the shift $\delta P$ and of the exit window on the shift $\delta A$ are, within experimental error, the same for cases 1 and 2 . This can only be explained when the first row and first column of the window-imperfection matrices are zero, which is true for a birefringent window.

On the basis of these conclusions the plane of incidence may be determined in the presence of birefringent windows as illustrated in fig. 3. The lines with slopes $m_{1}, m_{2}, m_{3}$ and $m_{4}$ in fig. 2 , determined in the presence of both windows, are

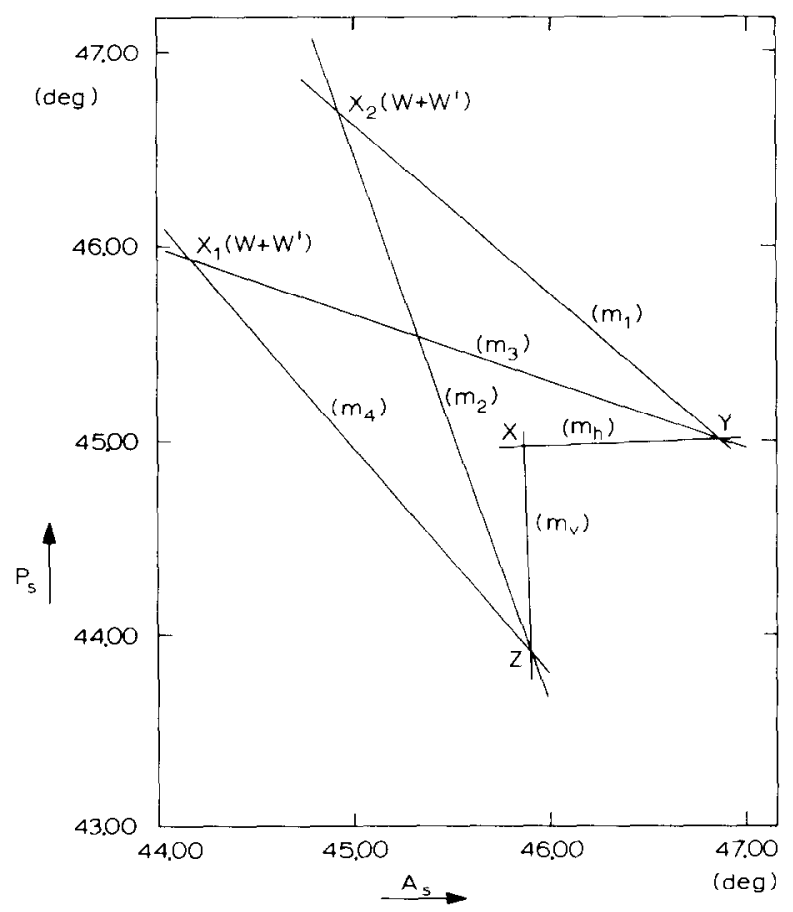

Fig. 3. Construction for determination of plane of incidence $\left(\mathrm{X}=P_{0}, A_{0}\right)$ in the presence of birefringent cell windows (see text). 
redrawn by reducing the $A$ values in fig. 2a and the $P$ values in fig. $2 \mathrm{~b}$ with $90^{\circ}$. The lines with slopes $m_{1}$ and $m_{3}$ intersect in point $\mathrm{Y}$, those with slopes $m_{2}$ and $m_{4}$ in $\mathrm{Z}$. The point $\mathrm{X}\left(P_{0}, A_{0}\right)$ indicates the plane of incidence $(P=0, A=0)$. The slopes of the lines $\mathrm{XY}$ and $\mathrm{XZ}$ are denoted by $m_{\mathrm{h}}$ and $m_{\mathrm{v}}$, the coordinates of $\mathrm{X}_{1}\left(W+W^{\prime}\right)$ and $\mathrm{X}_{2}\left(W+W^{\prime}\right)$ by $\left(P_{1}, A_{1}\right)$ and $\left(P_{2}, A_{2}\right)$. From the above mentioned conclusions it follows that

$m_{\mathrm{h}}=\left(m_{1} m_{4}-m_{2} m_{3}\right) /\left(m_{4}-m_{3}+m_{1}-m_{2}\right)$,

$m_{\mathrm{V}}=\left[m_{1} m_{3}\left(m_{2}-m_{4}\right)-m_{2} m_{4}\left(m_{1}-m_{3}\right)\right] /\left(m_{2} m_{3}-m_{1} m_{4}\right)$.

According to eqs. (5) and (6)

$m_{1}=1 / m_{4}, \quad m_{2}-1 / m_{3}$.

This results in $m_{\mathrm{h}}=0$ (a horizontal line) and $m_{\mathrm{v}}=\infty$ (a vertical line), which gives for the coordinates of $\mathrm{X}$ :

$P_{0}=P_{1}+\left\{\left(P_{2}-P_{1}\right)-\bar{m}_{1}\left(A_{2}-A_{1}\right)\right\} /\left(1-\bar{m}_{1} \bar{m}_{2}\right)$,

$A_{0}=A_{1}+\left\{\left(P_{2}-P_{1}\right)-\bar{m}_{2}\left(A_{2}-A_{1}\right)\right\} /\left(1-\bar{m}_{4} \bar{m}_{2}\right)$.

The averaged line slopes

$\bar{m}_{1}=1 / \bar{m}_{4}=\frac{1}{2}\left(m_{1}+1 / m_{4}\right), \quad \bar{m}_{2}=1 / \bar{m}_{3}=\frac{1}{2}\left(m_{1}+1 / m_{3}\right)$

are used to eliminate the experimental errors. Table 6 presents the values of $P_{0}$ and $A_{0}$ calculated with eqs. (20) and (21) for the three samples. The results appear to be in good agreement with the coordinates of $X_{1}$ and $X_{2}$ determined graphically in the absence of the windows, they are also given in table 6 .

The success of determining the actual reference positions $P_{0}$ and $A_{0}$ without removing the windows depends on the values of the ellipsometric parameters $\Delta$ and $\psi$, which determine the slopes $m$. Small differences between $m_{1}$ and $m_{3}$ cq. $m_{2}$ and $m_{1}$ give rise to large uncertainties in the point of intersection $\mathrm{Y}$ cq. $\mathrm{Z}$.

The elements $B_{W}$ and $B_{W}$ of the window imperfection matrices can be calculated with eqs. (9a) and $(9 \mathrm{~b})$. The shifts $\delta P\left(W+W^{\prime}\right)$ and $\delta A\left(W+W^{\prime}\right)$ are determined with a construction as in fig. 3 . Small deviations of $\Delta$ and $\psi$ have no

Table 7

The averaged values of the elements of the cell-window imperfection matrices calculated from different measuring procedures

\begin{tabular}{lllll}
\hline Procedure & $B_{W}$ & $A_{W}$ & $B_{W^{\prime}}$ & $A_{W^{\prime}}$ \\
\hline$P S A$ in ex tinction & -0.022 & & 0.023 & \\
$P S A_{\text {rot }}$ ellipsometer & -0.022 & -0.003 & 0.025 & -0.007 \\
$P S A_{\text {rot }^{A}}$ ellipsometer & -0.022 & -0.001 & 0.024 & -0.007 \\
\hline
\end{tabular}


influence on the results, so that $\Delta$ and $\psi$ values without window corrections can be used. The results are given in table 7 .

\subsection{Window influences in rotating-analyzer ellipsometers}

In section 4.1 the determination of the elements $B_{k}$ of the window imperfection matrices in the presence of windows has been described. To determine both elements $A_{k}$ and $B_{k}$ with our rotating-analyzer ellipsometers, measurements have been carried out with and without windows.

With the intensity ellipsometer $B_{W}$ can be determined directly by substituting the Fourier coefficient $b$ measured in the calibration modes, the other elements follow from the shift in the Fourier coefficients caused by introducing a window in the measuring modes. The Fourier coefficients are given in table 4 . With the phase ellipsometer $B_{W}$ may be calculated directly by substituting $T_{2}$ or $T_{4}$, determined in the calibration modes, in eq. (15). For a pair of zones the other elements can be calculated by combining the shift in $T_{2}$ or $T_{4}$ caused by introducing a window in the measuring modes, $T_{2}$ and $T_{4}$ are given in table 5 . Calculations of the elements $A_{k}$ for zone pairs $(3,4)$ and $(7,8)$ have been avoided, because the expressions for $A_{W}$ and $A_{W^{\prime}}$ also contain $B_{W^{\prime}}$, which in our case is relatively large.

The results, given in table 7 , have been obtained by averaging over the different modes and the three samples. Within experimental error $( \pm 0.002)$ they are the same for the different measuring procedures.

\section{Conclusions}

The plane of incidence can be determined in the presence of cell windows with small retardation by using a graphical method illustrated in fig. 3 .

As with the $P C S A$ configuration, in the $P S A_{\text {rot }}$ and $P S A_{\text {rot }} A$ rotating-analyzer ellipsometers, it is also impossible to correct for window birefringence by averaging over the different measuring modes. The corrections can be achieved by using the Mueller-imperfection matrices whose elements can be determined from measurements with and without windows.

\section{References}

[1] R.H. Muller, Surface Sci. 56 (1976) 19.

[2] P.S. Hauge and F.H. Dill, IBM J. Res. Develop. 17 (1973) 472.

[3] D.E. Aspnes and A.A. Studna, Appl. Opt. 14 (1975) 220.

[4] R.W. Stobie, B. Rao and M.J. Dignam, Appl. Opt. 14 (1975) 999.

[5] R.M.A. Azzam and N.M. Bashara, Ellipsometry and Polarized Light (North-Holland, Amsterdam, 1977).

[6] R.M.A. Azzam and N.M. Bashara, J. Opt. Soc. Am. 64 (1974) 1459. 
[7] Y.J. van der Meulen and N.C. Hien, J. Opt. Soc. Am. 64 (1974) 804.

[8] R.W. Stobie, B. Rao and M.J. Dignam, J. Opt. Soc. Am. 65 (1975) 25.

[9] D.E. Aspnes, J. Opt. Soc. Am. 64 (1974) 812.

[10] F.L. McCrackin, E. Passaglia, R.K. Stromberg and H.L. Steinberg, J. Kes. Natl. Bur. Std. 67A (1963) 363.

[11] F,L. McCrackin, J. Opt. Soc. Am. 60 (1970) 57.

[12] R.M.A. Az7.am and N.M. Bashara, J. Opt. Soc. Am. 61 (1971) 1380.

[13] W. Budde, Appl. Opt. 1 (1962) 201.

\section{Discussion}

V.M. Bermudez (Naval Research Laboratory): I have two questions. First, have you tried annealing your cell windows? We have found that low-strain fused-silica windows can be produced by (following the suggestions of S.E. Schnatterly) first fusing the window to a section of silica tube, then annealing this piece and fusing it to a graded seal which previously had been attached to a UHV flange. Retardations as small as $0.3^{\circ}$ at $\lambda=300 \mathrm{~nm}$ can be obtained. In the case of Pyrex, it should be possible to anneal the entire window assembly after fabrication. Second, have you tried to reduce the magnitude of the systematic errors by suitably orienting the principle axes of the windows with respect to the plane of incidence? For the case of the polarization-modulation ellipsometer, the effect of exit-window bircfringence can be climinated by orienting the principal axis of the exit window at $45^{\circ}$ to the plane of incidence. However, systematic correction is still required for the entrance-window birefringence.

L.J. Hanekamp: It is our experience that also annealing of pyrex cell windows can reduce the birefringence considerably. However, conventional UHV cell windows show birefringence caused by mechanical strains upon mounting and upon baking of the UHV system. Being interested in the experimental determination of the effect of the birefringence, we oriented our windows experimentally in a position with maximum influence of the birefringence on $\Delta$ and $\psi$.

D.E. Aspnes (Bell Laboratories): How do the windows in this system respond to the usual cross-polarizer strain birefringence test?

L.J. Hanekamp: Between cross-polarizers the windows showed slight birefringence, which increased from the center of the windows to the edge. 